\title{
CITORREDUCCIÓN ÓPTIMA EN CÁNCER DE OVARIO AVANZADO TRATADO CON PACLITAXEL A DOSIS DENSA Y CARBOPLATINO SEGUIDO DE CIRUGÍA DE INTERVALO EN EL INSTITUTO NACIONAL DE ENFERMEDADES NEOPLÁSICAS DEL PERÚ
}

\author{
Cindy Alcarraz',a, Johana Muñiz ${ }^{1, b}$, Luis Mas ${ }^{1, a}$, Mivael Olivera1,a, \\ Zaida Morante ${ }^{1, a}$, Manuel Alvarez ${ }^{1, c}$, Raul Mantilla ${ }^{1, d}$, Jhajaira Araujo,e, Joseph Pinto ${ }^{2, e}$
}

\begin{abstract}
RESUMEN
Objetivos. Determinar la tasa de citorreducción óptima en pacientes con cáncer de ovario avanzado que recibieron quimioterapia neoadyuvante con carboplatino y paclitaxel dosis densa seguido de cirugía de citorreducción de intervalo $(\mathrm{CCl})$. Materiales y métodos. Estudio de una serie de casos retrospectiva de mujeres peruanas tratadas con quimioterapia neoadyuvante con carboplatino (AUC $6 \mathrm{mg} / \mathrm{ml} / \mathrm{min}$ ) y paclitaxel $\left(80 \mathrm{mg} / \mathrm{m}^{2}\right.$ semanal) seguido de $\mathrm{CCl}$, en el Instituto Nacional de Enfermedades Neoplásicas durante el período 2010-2014. Resultados. Los 41 pacientes que alcanzaron cirugía de intervalo, tuvieron una mediana de edad de 59 años (rango: 47-73 años). En 37 (90,2\%) pacientes se reportó histología de adenocarcinoma seroso de alto grado. Treinta y cuatro $(82,9 \%)$ lograron citorreducción óptima y cinco $(14,7 \%)$ respuesta patológica completa. La sobrevida libre de progresión al año y 2 años fueron 74,7\% y 51,8\%, respectivamente. La sobrevida global al año y 2 años fue $85,2 \%$ y $71,4 \%$, respectivamente. El riesgo de progresión y muerte fue mayor en pacientes sin citorreducción óptima y pacientes con niveles de dosaje del antígeno carcinoembrionario 125 postoperatorio > $30 \mathrm{U} / \mathrm{ml}$. Conclusiones. La neoadyuvancia con carboplatino y paclitaxel dosis densa logró una frecuencia elevada de citorreducción óptima. Los niveles de antígeno carcinoembrionario 125 postoperatorios y citorreducción óptima resultaron factores independientes de sobrevida libre de progresión y sobrevida global.
\end{abstract}

Palabras clave: Neoplasias ováricas; Quimioterapia; Terapia neoadyuvante; Procedimientos quirúrgicos de citorreducción; Perú. (fuente: DeCS BIREME).

\section{OPTIMAL CYTOREDUCTION IN ADVANCED OVARIAN CANCER TREATED WITH DOSE-DENSE PACLITAXEL AND CARBOPLATIN FOLLOWED BY INTERVAL SURGERY AT THE PERUVIAN NATIONAL INSTITUTE OF NEOPLASTIC DISEASES}

\begin{abstract}
Objectives. To determine the rate of optimal cytoreduction in patients with advanced ovarian cancer who received neoadjuvant chemotherapy with dose-dense carboplatin and paclitaxel followed by interval debulking surgery (IDS). Materials and Methods. A retrospective study of a series of cases of Peruvian women treated with neoadjuvant chemotherapy with carboplatin (6 AUC $\mathrm{mg} / \mathrm{mL} / \mathrm{min}$ ) and paclitaxel $\left(80 \mathrm{mg} / \mathrm{m}^{2}\right.$ weekly) followed by IDS, at the National Institute of Neoplastic Diseases during the 2010-2014 period. Results. The 41 patients who made it to the interval surgery had a median age of 59 years (range: $47-73$ years). In 37 (90.2\%) patients, high-grade serous adenocarcinoma histology was reported. Thirty-four $(82.9 \%)$ achieved optimal cytoreduction and five (14.7\%), a complete pathological response. Progression-free survival at one year and two years was $74.7 \%$ and $51.8 \%$, respectively. Overall survival at one year and two years was $85.2 \%$ and $71.4 \%$, respectively. The risk of progression and death was greater in patients without optimal cytoreduction and in patients with postsurgery levels of carcinoembryonic antigen $125>30 \mathrm{U} / \mathrm{mL}$. Conclusions. Neoadjuvant therapy with dose-dense carboplatin and paclitaxel achieved an elevated frequency of optimal cytoreduction. The post-surgery levels of carcinoembryonic antigen 125 and optimal cytoreduction were independent factors of progression-free survival and overall survival.
\end{abstract}

Keywords: Ovarian neoplasms, Chemotherapy, Neoadjuvant therapy, Cytoreduction surgical procedures, Peru (source: MeSH NLM).

\footnotetext{
Instituto Nacional de Enfermedades Neoplásicas. Lima, Perú.

Oncosalud, AUNA. Lima, Perú.

Médico oncólogo; ${ }^{\mathrm{b}}$ médico residente de oncología; ${ }^{\mathrm{c}}$ médico cirujano ginecólogo oncólogo; ${ }^{\mathrm{d}}$ estadístico; ${ }^{\mathrm{e}}$ biólogo

Recibido: 15/04/2017 Aprobado: 07/03/2018 En línea: 05/04/2018
}

Citar como: Alcarraz C, Muñiz JG, Mas L, Olivera M, Morante Z, Alvarez M, et al. Citorreducción óptima en cáncer de ovario avanzado tratado con paclitaxel a dosis densa y carboplatino seguido de cirugía de intervalo en el Instituto Nacional de Enfermedades Neoplásicas del Perú. Rev Peru Med Exp Salud Publica. 2018;35(1):46-54. doi: 10.17843/rpmesp.2018.351.3599. 


\section{INTRODUCCIÓN}

El cáncer de ovario es la principal causa de muerte debido a cáncer ginecológico en Europa y Estados Unidos ${ }^{(1,2)}$. En 2018, la tasa de incidencia a nivel mundial es de 6,3 por 100000 mujeres en países desarrollados y 9,3 por 100 000 mujeres en países en desarrollo; en Europa alcanza una tasa de incidencia de 10,1 por 100000 mujeres y en Norte América de 8,7 por 100000 mujeres, mientras que en América del Sur es 6,2 por $100000^{(3)}$.

El cáncer de ovario es el segundo cáncer ginecológico más común en los países en desarrollo, representa el 18,8\% de todas las neoplasias ginecológicas y el $28,7 \%$ en los países desarrollados (4); la tasa de letalidad es de $54,8 \%$ y $59,2 \%$ en países en desarrollo y desarrollados, respectivamente. La elevada tasa de mortalidad se debe principalmente al diagnóstico en estadios avanzados ${ }^{(5)}$. En Perú según el registro de Lima Metropolitana 2004-2005, el cáncer de ovario es la octava neoplasia más frecuente y la segunda neoplasia ginecológica después del cáncer de cuello uterino, representando la segunda causa de muerte debido a una neoplasia ginecológica ${ }^{(6)}$. EI INEN registró 143094 casos nuevos de cáncer entre el 2000 y 2014 de los cuales 89717 fueron mujeres, los casos nuevos de cáncer de ovario fueron $2840(3,2 \%)^{(7)}$.

Aproximadamente el $75 \%$ de los pacientes con cáncer de ovario son diagnosticados en estadios avanzados, y presenta una sobrevida menor al $30 \%$ a los cinco años ${ }^{\left({ }^{(8)}\right.}$. Existe una fuerte asociación entre la enfermedad residual postoperatoria y la supervivencia en cáncer de ovario epitelial ${ }^{(8,9)}$. En ese sentido, los pacientes con enfermedad residual $<1 \mathrm{~cm}$ (citorreducción óptima) presentan mejor sobrevida que aquellos con enfermedad $>1 \mathrm{~cm}$ (citorreducción subóptima). Por ello, la citorreducción óptima es un factor pronóstico independiente de sobrevida ${ }^{(10)}$.

En la actualidad, el tratamiento estándar para el cáncer de ovarioavanzado es la combinación de cirugía de citorreducción primaria (CCP) seguido de quimioterapia adyuvante con paclitaxel a dosis densa $\left(80 \mathrm{mg} / \mathrm{m}^{2}\right)$ y carboplatino (6 AUC), esta asociación ha demostrado mejorar la sobrevida libre de progresión (SLP) y la sobrevida global (SG) en pacientes con cáncer de ovario estadio II-IV, comparado con el esquema convencional de paclitaxel $\left(175 \mathrm{mg} / \mathrm{m}^{2}\right)$ y carboplatino (6 AUC) cada tres semanas ${ }^{(11,12)}$. El uso racional de paclitaxel a dosis densa, radica en estudios preclínicos, donde se observó que el tiempo de exposición a la droga, es determinante de su efecto citotóxico; mientras que dosis bajas en intervalos cortos inducen apoptosis e inhibe la angiogénesis en células tumorales ${ }^{(13,14)}$.

Lamentablemente, algunos pacientes presentanenfermedad irresecable o no se encuentran aptos para CCP debido a un estado funcional deficiente. Para este grupo de pacientes,

\section{MENSAJES CLAVE}

Motivación para realizar el estudio. Los pacientes con cáncer ovario avanzado tienen un pobre pronóstico siendo necesario determinar las alternativas de manejo apropiadas para conseguir una cirugía óptima y control de la enfermedad.

Principales hallazgos. La quimioterapia neoadyuvante con carboplatino y paclitaxel dosis densa seguido de cirugía de citorreducción de intervalo es una estrategia para lograr citorreducción óptima en pacientes con cáncer de ovario avanzado.

Implicancias. Con el uso de quimioterapia neoadyuvante seguido de cirugía de citorreducción se podrá mejorar la sobrevida en este grupo de pacientes con cáncer de ovario avanzado.

el tratamiento neoadyuvante con quimioterapia resulta una estrategia para lograr una cirugía de citorreducción óptima, conocida como cirugía de citorreducción de intervalo (CCI).

La quimioterapia neoadyuvante a dosis convencionales con paclitaxel $\left(175 \mathrm{mg} / \mathrm{m}^{2}\right)$ y carboplatino (AUC 6) cada tres semanas, seguido de $\mathrm{CCl}$, ha demostrado tasas altas de CO con SLP y SG no inferiores a la CCP ${ }^{(15-17)}$. Además, la quimioterapia neoadyuvante seguida de $\mathrm{CCl}$ es más segura y condiciona mejores resultados postoperatorios ${ }^{(18)}$. Mientras que los resultados del esquema con paclitaxel dosis densa en el escenario neoadyuvante no han sido comparados con el convencional en forma prospectiva y comparativa.

En el Instituto Nacional de Enfermedades Neoplásicas (INEN), el manejo del cáncer ovario es basado en la evidencia sustentada en la guía de práctica clínica institucional y la guía de la National Cancer Comprehensive Network de los Estados unidos (NCCN). La primera considera el esquema de carboplatino y paclitaxel dosis densa en el manejo sistémico de la enfermedad avanzada (19) y la segunda propone quimioterapia neoadyuvante con alta calidad de evidencia para pacientes con cáncer de próstata en estadios clínicos III-IV con enfermedad de masa (Bulky) o no candidato a cirugía ${ }^{(20)}$. Sin embargo, hasta la fecha no tenemos una evaluación institucional de los resultados de la quimioterapia neoadyuvante que se viene aplicando en este grupo de pacientes.

Por ello, el objetivo de nuestro estudio fue determinar la frecuencia de citorreducción óptima en pacientes con cáncer de ovario avanzado que recibieron quimioterapia neoadyuvante con carboplatino y paclitaxel a dosis densa seguido de cirugía de citorreducción de intervalo en el INEN.

\section{MATERIALES Y MÉTODOS}

\section{DISEÑO Y POBLACIÓN DEL ESTUDIO}

Se realizó un estudio observacional de una serie de casos retrospectiva en pacientes con diagnóstico de cáncer de 
ovario tratadas en el INEN entre enero del 2010 a diciembre de 2014.

EI INEN es el centro oncológico de referencia a nivel nacional. El manejo del cáncer de ovario avanzado en el INEN es multidisciplinario, el gineco-oncólogo determina la resecabilidad de la enfermedad, y coordina con el departamento de medicina oncológica el tratamiento sistémico, cuando la citorreducción óptima no puede garantizarse o las comorbilidades y/o estado funcional de paciente dificultan el abordaje quirúrgico.

\section{CRITERIOS DE SELECCIÓN}

Se incluyeron pacientes de 14 años de edad o más, con confirmación histológica de cáncer de ovario epitelial avanzado, con estadios clínicos IIIC y IV según clasificación de la Federación de Ginecólogos y Obstetras (FIGO) y que recibieron quimioterapia neoadyuvante a base de paclitaxel a dosis densa y carboplatino. Se excluyeron pacientes con cáncer de ovario epitelial avanzado sometidas a cirugía de citorreducción primaria o con tratamiento con quimioterapia neoadyuvante a dosis convencionales o aquellas pacientes que recibieron quimioterapia en otra institución.

\section{VARIABLES}

La información clínica referente a las características preoperatorias se obtuvo de la historia clínica, se incluyó la edad al diagnóstico, estadio clínico (EC), estado funcional de acuerdo a la escala de Eastern Cooperative Oncology Group (ECOG), tipo histológico, el tamaño tumoral en centímetros previo al tratamiento neoadyuvante, el dosaje del antígeno carcinoembrionario 125 (CA125) al momento del diagnóstico y después de la cirugía, la albúmina y la creatinina sérica de inicio.

Las características del tratamiento que se consideraron fueron: el número de cursos del tratamiento neoadyuvante, tasa de citorreducción, el tiempo entre el final de la neoadyuvancia y la citorreducción.

Las características patológicas fueron: el tamaño en centímetros $(\mathrm{cm})$ del tumor residual, la tasa de respuesta patológica completa (enfermedad residual no viable después de la cirugía), citorreducción óptima (enfermedad residual menor de $1 \mathrm{~cm}$ ) y citorreducción subóptima (enfermedad residual $>1 \mathrm{~cm}$ ).

Además, se registró la presencia y grados de toxicidades hematológicas (anemia, neutropenia y plaquetopenia) y no hematológicas (gastrointestinales, neurológicas y renales), de acuerdo a los Criterios Terminológicos Comunes para Eventos Adversos (CTCAE v. 4) del departamento de Salud y Servicios Humanos de los Estados Unidos del Instituto Nacional del Cáncer, $2009^{(21)}$.

\section{ANÁLISIS ESTADÍSTICO}

Se realizó un análisis descriptivo de las variables en estudio, a través de frecuencias, porcentajes y medidas resumen. Las diferencias entre los grupos de pacientes con y sin citorreducción óptima respecto a características cuantitativas medidas al diagnóstico, se evaluaron con la prueba t para muestras independientes o su correspondiente prueba no paramétrica (previa evaluación de normalidad a través de la prueba de Shapiro-Wilk). Asociaciones entre el nivel de respuesta patológica con características cualitativas al diagnóstico, fueron evaluados con la prueba Chi-cuadrado, aplicando la corrección de Yates cuando fue necesario.

Para la estimación de la sobrevida global (SG), el tiempo de seguimiento se calculó desde la fecha del diagnóstico (definida como la fecha de la primera confirmación patológica de la neoplasia maligna obtenida del líquido pleural/peritoneal, aspiración con aguja fina o biopsia core del tumor sólido) hasta la fecha de muerte (evento de interés) o fecha de último control. Para la estimación de la sobrevida libre de progresión (SLP), el seguimiento se calculó desde la fecha de diagnóstico hasta la fecha de progresión o recurrencia (evento de interés, considerado como un aumento del CA125 y/o evidencia radiológica de progresión) o la fecha de muerte o la fecha de último control. Los pacientes que no presentaron el evento de interés fueron considerados como censurados. Las curvas de sobrevida se estimaron con el método de Kaplan-Meier y diferencias en las sobrevidas según el resultado de la citorreducción (óptima o subóptima) se evaluaron con la prueba log-rank. El valor pronóstico de la citorreducción óptima se analizó con un modelo multivariado de riesgos proporcionales de Cox. Un valor $p<0,05$ se consideró para una asociación o significativa. Se utilizó el programa R (R Core Team 2017. R Foundation for Statistical Computing, Vienna, Austria. URL https://www.R-project.org/).

\section{CONSIDERACIONES ÉTICAS}

El protocolo de este estudio fue aprobado por el Comité Institucional de Ética del Instituto Nacional de Enfermedades Neoplásicas. Se mantuvo la confidencialidad de la información obtenida de las historias clínicas durante todo el tiempo de ejecución de este estudio, asignando un código de identificación a los casos incluidos, sólo el investigador principal tuvo conocimiento de la codificación asignada a cada paciente.

\section{RESULTADOS}

Durante los años 2010-2014, 335 pacientes presentaron cáncer de ovario avanzado de tipo epitelial estadios clínicos III C y IV, de los cuales 116 no recibieron tratamiento en el INEN, 152 pacientes recibieron cirugía de citorreducción 
primaria 50 recibieron quimioterapia neoadyuvante a dosis densa y 17 recibieron quimioterapia a dosis convencionales.

De los 50 pacientes con diagnóstico de cáncer de ovario tipo epitelial estadio clínico (EC) IIIC y IV que recibió quimioterapia neoadyuvante con carboplatino y paclitaxel a dosis densa, el $82,0 \%(n=41)$ fue sometido a $\mathrm{CCl}$ y el $18,0 \%(n=9)$ no alcanzó $\mathrm{CCl}$, ocho debido a progresión de enfermedad y uno por obstrucción intestinal posterior a quimioterapia.

La mediana de edad al diagnóstico de los 41 pacientes que recibieron tratamiento neoadyuvante con quimioterapia seguido de $\mathrm{CCl}$ fue de 59 años, rango de 42 a 73 años. Presentaron enfermedad EC IIIC 63,4\% $(n=26)$ y EC IV $36,6 \%(n=15)$. El $63,4 \%$ presentó estado funcional ECOG 1 ( $n=26), 34,1 \%$ ECOG $2(n=14)$ y $2,4 \%$ ECOG $3(n=1)$. El tipo histológico más frecuente fue el adenocarcinoma seroso presente en el 90,2 \% $(n=37)$ de pacientes. El tamaño tumoral al diagnóstico fue evaluable en 28 pacientes, presentando una mediana de $10 \mathrm{~cm}$ [rango: 5 a $20 \mathrm{~cm}$. La mediana del nivel de CA125 al diagnóstico fue de $1069 \mathrm{U} / \mathrm{ml}$ [rango: 42,95 a $15999 \mathrm{U} / \mathrm{ml}$ ]; la mediana de los valores de creatinina sérica fue de $53 \mathrm{mg} / \mathrm{dL}$ [rango: 23 a $97 \mathrm{mg} / \mathrm{dL}$ ] y de los valores de albúmina sérica fue de 3,56 g/dL [rango: 2 a 4,8 g/dL]. El 39,0 \% $(n=16)$ de pacientes recibió tres cursos de tratamiento neoadyuvante, el 4,9\% $(n=2)$ recibió cuatro cursos y el $56,1 \%(n=23)$ recibió seis cursos (Tabla 1).

Tabla 1. Características clínicas de los pacientes que recibieron quimioterapia neoadyuvante dosis densa y lograron cirugía de citorreducción de intervalo

\begin{tabular}{lc}
\hline Características & Frecuencia (\%) \\
\hline Edad en años & $59[42-73]^{*}$ \\
\hline Estadio & \\
\hline IIIC & $26(63,4)$ \\
\hline IV & $15(36,6)$ \\
\hline Estado funcional según escala ECOG & \\
$\quad$ uno & $26(63,4)$ \\
$\quad$ dos & $14(34,1)$ \\
$\quad$ tres & $1(2,4)$ \\
\hline Tipo histológico & \\
\hline Adenocarcinoma seroso & $37(90,2)$ \\
\hline Otros & $4(9,6)$ \\
\hline Tamaño tumoral (cm) & $10[5-20]^{*}$ \\
CA-125, (U/mL) & $1069[42,95-15999]^{*}$ \\
Creatinina (mg/dL) & $53[23-97]^{*}$ \\
\hline Albúmina (gr/dL) & $3,56[2-4,8]^{*}$ \\
\hline Cursos de neoadyuvancia & \\
\hline tres & $16(39,0)$ \\
\hline cuatro & $2(4,9)$ \\
\hline seis & $23(56,1)$ \\
\hline
\end{tabular}

ECOG: Eastern Cooperative Oncology Group *Mediana [rango]
De los 41 pacientes que lograron cirugía de citorreducción de intervalo, el 82,9\% $(n=34)$ logro citorreducción óptima, de los cuales el $14,7 \%(n=5)$ tuvieron respuesta patológica completa y el $85,3 \%(n=29)$ tuvo enfermedad residual menor de $1 \mathrm{~cm}$.

No se encontró una diferencia significativa entre los pacientes que lograron o no citorreducción óptima respecto a la edad, el tamaño tumoral antes del tratamiento neoadyuvante, el CA125 al momento del diagnóstico y la albúmina. Si hubo una diferencia significativa respecto a la creatinina, los pacientes que no lograron una citorreducción óptima tuvieron un nivel promedio de creatinina mayor que los pacientes que si lograron citorreducción óptima $(65,6$ $\mathrm{mg} / \mathrm{dL}$ vs $54,4 \mathrm{mg} / \mathrm{dL}, \mathrm{p}=0,045)$. La mediana del nivel de CA125 después de la cirugía fue de 19,83 [rango: 0 a $1825 \mathrm{U} / \mathrm{ml}$ ], esto evaluado en 31 pacientes. Se encontró una diferencia significativa en los valores de CA125 al diagnóstico y después de la cirugía $(p<0,001)$.

El $25,0 \%(n=4)$ y $75,0 \%(n=12)$ de los que recibieron tres cursos de tratamiento neoadyuvante con quimioterapia alcanzaron citorreduccion subóptima y óptima, respectivamente; el 12,0\% $(n=3)$ y el $88,0 \%(n=22)$ de los que recibieron más de tres cursos de tratamiento neoadyuvante con quimioterapia, alcanzaron citorreducción subóptima y óptima, respectivamente. No se encontró asociación significativa entre el número de cursos de tratamiento neoadyuvante y el nivel de respuesta patológica $(p=0,513)$.

La mediana del tiempo de observación para la estimación de la sobrevida libre de progresión, de los pacientes que lograron cirugía de citorreducción de intervalo, fue de 17 meses y su sobrevida libre de progresión a los 12 , 24 y 36 meses se estimó en $74,7 \%, 51,8 \%$ y 35,6 \%, respectivamente. La mediana del tiempo de sobrevida libre de progresión se estimó en 25 meses. En los pacientes que lograron citorreducción óptima, la sobrevida libre de progresión se estimó a los 12, 24 y 36 meses en 81,9\%, $59,2 \%$ y $40,7 \%$, respectivamente, y en los que no tuvieron citorreducción óptima fue $35,7 \%$ y $0,0 \%$ a los 12 y 17 meses, respectivamente; encontrándose una diferencia significativa $(p=0,002)$ (Figura 1).

La mediana del tiempo de observación para la estimación de la sobrevida global de los pacientes que lograron cirugía de citorreducción de intervalo, fue de 21 meses y su sobrevida global a los 12, 24 y 36 meses se estimó en $85,2 \%, 71,4 \%$ y $66,3 \%$, respectivamente; no fue posible determinar la mediana del tiempo de sobrevida global porque la curva no se ubicó por debajo del $50 \%$ de la probabilidad acumulativa. En los pacientes que lograron citorreducción óptima, la sobrevida global se estimó a los 12 , 24 y 36 meses en $91,1 \%, 80,8 \%$ y 75,0 \%, respectivamente, y en los que no tuvieron citorreducción óptima se estimó en $57,1 \%$ a los 12 meses; encontrándose una diferencia significativa $(p=0,002)$ (Figura 2). 


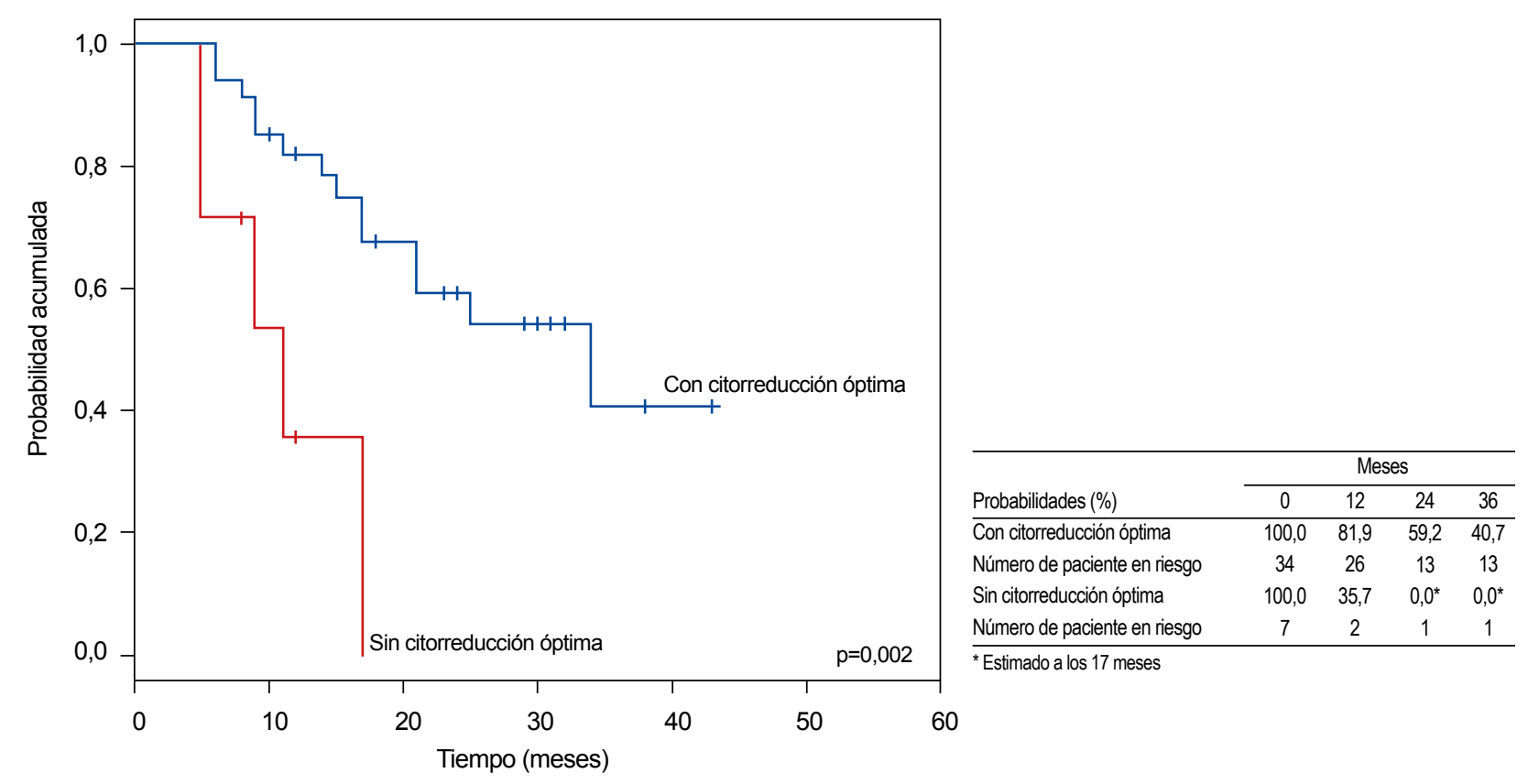

Figura 1. Curvas estimadas de la sobrevida libre de progresión según estado de citorreducción óptima en pacientes que recibieron quimioterapia neoadyuvante dosis densa y lograron cirugía de citorreducción de intervalo.

Al evaluar la citorreducción óptima como factor pronóstico ajustado por el nivel de creatinina, se encontró que los pacientes que no alcanzaron citorreducción óptima mostraron mayor riesgo de progresión (HRa=5,58; IC $95 \%$ : 1,80-17,28) y muerte (HRa=5,53; IC $95 \%$ : 1,59-19,25); respecto a los que lograron citorreducción óptima.
Asimismo, se encontró que los pacientes sometidos a quimioterapia neoadyuvante y cirugía de intervalo, con un nivel postoperatorio de CA125 >30 U/mL mostraron mayor riesgo de progresión ( $\mathrm{HR}=3,02$; IC $95 \%$ : 1,02-8,93) y muerte (HR=4,57; IC $95 \%$ : 1,14-18,34); que los pacientes con nivel de CA125 $\leq 30 \mathrm{U} / \mathrm{mL}$ (Figura 3 y 4 ).

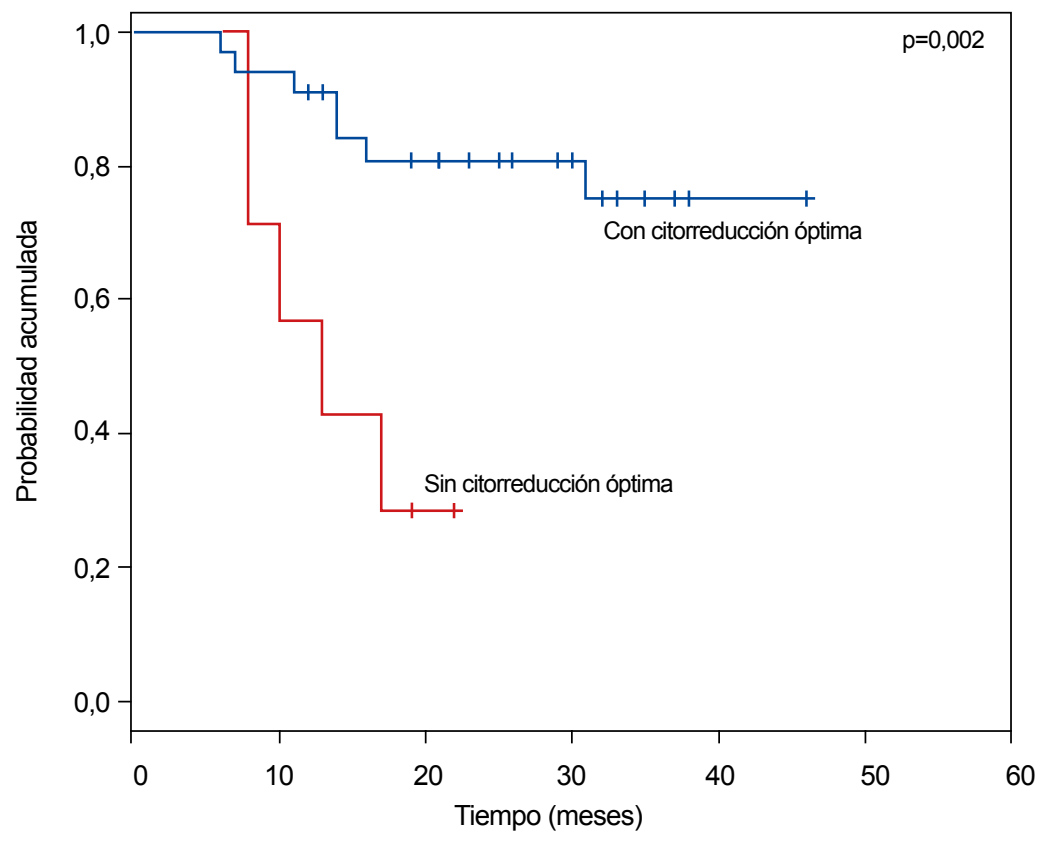

\begin{tabular}{lcccc}
\hline & \multicolumn{4}{c}{ Meses } \\
\cline { 2 - 5 } Probabilidades (\%) & 0 & 12 & 24 & 36 \\
\hline Con citorreducción óptima & 100,0 & 91,1 & 80,8 & 75,0 \\
Número de paciente en riesgo & 34 & 29 & 18 & 4 \\
Sin citorreducción óptima & 100,0 & 57,1 & & \\
Número de paciente en riesgo & 7 & 4 & & \\
\hline
\end{tabular}

Figura 2. Curvas estimadas de la sobrevida global según citorreducción óptima en pacientes que recibieron quimioterapia neoadyuvante a dosis densa y lograron cirugía de citorreducción de intervalo 


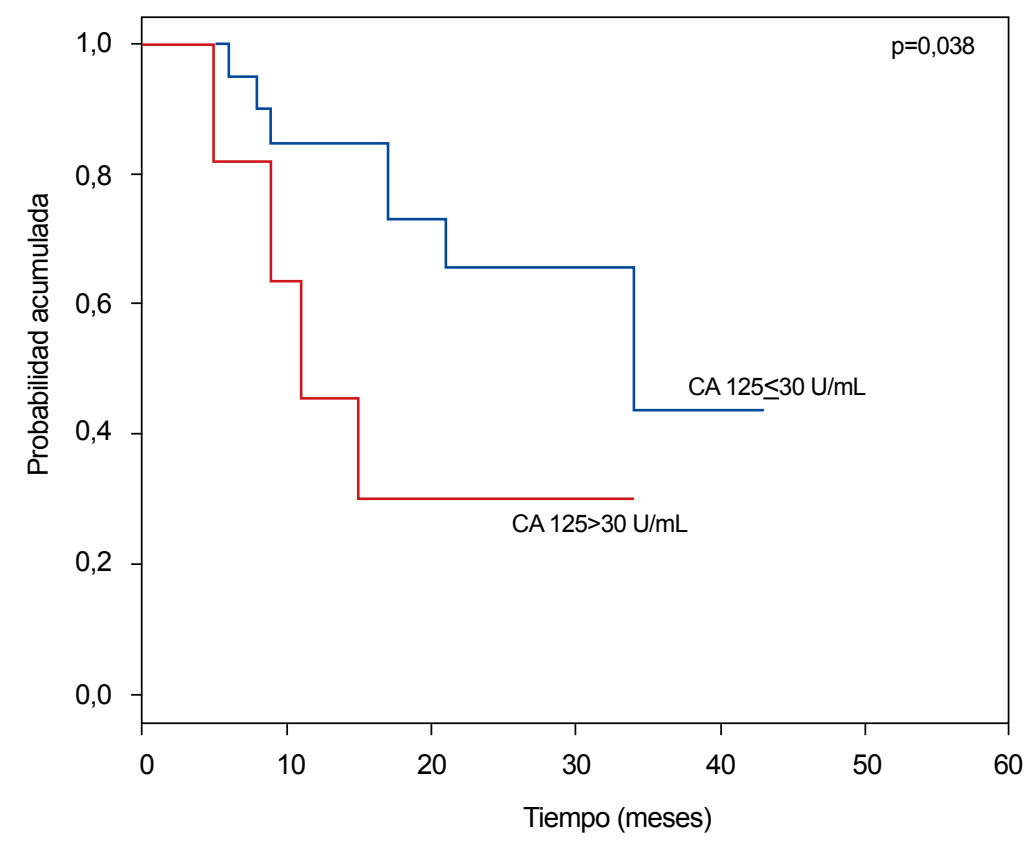

\begin{tabular}{lcccc}
\hline & \multicolumn{4}{c}{ Meses } \\
\cline { 2 - 5 } Probabilidades (\%) & 0 & 12 & 24 & 36 \\
\hline CA 125 $\leq 30 \mathrm{U} / \mathrm{mL}$ & 100,0 & 85,0 & 65,6 & 43,7 \\
Número de paciente en riesgo & 20 & 16 & 8 & 2 \\
CA 125>30 U/mL & 100,0 & 45,5 & 30,3 & \\
Número de paciente en riesgo & 11 & 5 & 1 & \\
\hline
\end{tabular}

Figura 3. Sobrevida libre de progresión según nivel de CA125 postoperatorio en pacientes sometidos a quimioterapia neoadyuvante a dosis densa que lograron cirugía de citoreducción de intervalo

CA125: antígeno carcinoembrionario 125

El 78,0\% $(n=39)$ de pacientes presentaron algún grado de toxicidad hematológica, el 8,0\% $(n=4)$ presentó anemia grado $3-4$, el $24,0 \%(n=12)$ neutropenia grado $3-4$ y el $6 \%(n=3)$ plaquetopenia grado 3 . Entre las toxicidades no hematológicas: el $4,0 \% \quad(n=2)$ presentó toxicidad gastrointestinal de grado 4 , el 16,0 \% (n=8) toxicidad neurológica de grado 1 y el $2,0 \%(n=1)$ toxicidad renal de grado 1 (Tabla 2). Un paciente falleció después del segundo curso de quimioterapia, debido a obstrucción intestinal.

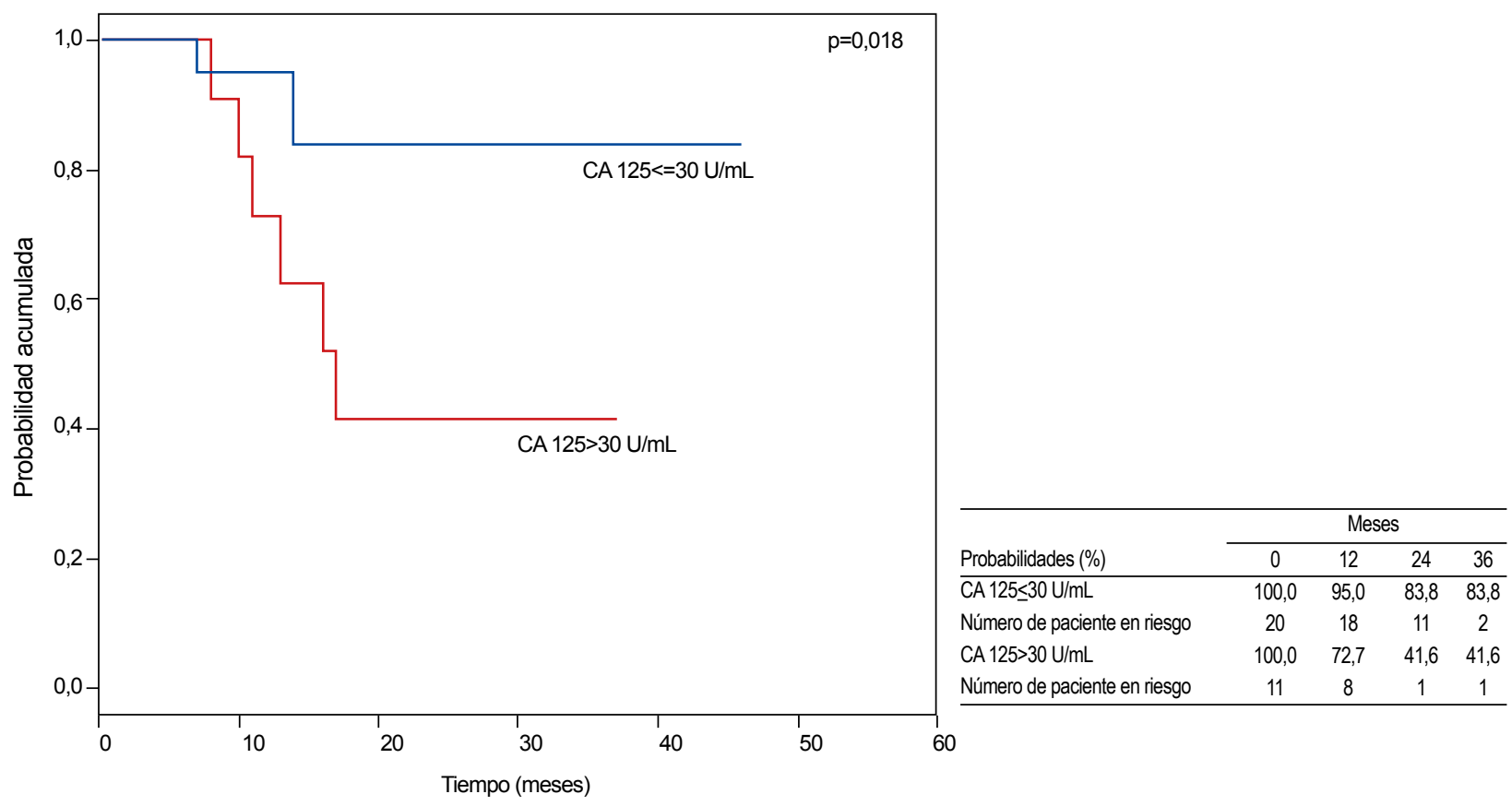

Figura 4. Sobrevida global según nivel de CA125 post operatorio en pacientes sometidos a quimioterapia neoadyuvante dosis densa que lograron cirugía de citorreducción de intervalo.

CA125:antígeno carcinoembrionario 125 
Tabla 2. Frecuencia, tipos y grados de toxicidad asociados a quimioterapia neoadyuvante a dosis densa según Criterios Terminológicos Comunes para Eventos Adversos (CTCAE V.4.)

\begin{tabular}{lrrrr}
\hline & \multicolumn{3}{c}{ Grado } \\
\cline { 2 - 5 } Toxicidad n (\%) & $\mathbf{1}$ & $\mathbf{2}$ & $\mathbf{3}$ & $\mathbf{4}$ \\
\hline Hematológicas & $13(26,0)$ & $13(26,0)$ & $3(6,0)$ & $1(2,0)$ \\
Anemia & $6(12,0)$ & $16(32,0)$ & $7(14,0)$ & $5(10,0)$ \\
Neutropenia & $4(8,0)$ & $9(18,0)$ & $3(6,0)$ & 0 \\
Plaquetopenia & $4(8,0)$ & $2(4,0)$ & 0 & $2(4,0)$ \\
No hematológicas & $8(16,0)$ & 0 & 0 & 0 \\
Gastrointestinal & $1(2,0)$ & 0 & 0 & 0 \\
Neurológico & & & & \\
Renal & & 0 & \\
\hline
\end{tabular}

\section{DISCUSIÓN}

El presente estudio evaluó la respuesta a la quimioterapia neoadyuvante con carboplatino y paclitaxel a dosis densa seguido de $\mathrm{CCl}$ en pacientes con cáncer de ovario epitelial avanzado, que generalmente no pueden ser sometidos a CCP por el deterioro del estado funcional al diagnóstico. Los pacientes con cáncer de ovario avanzado que recibieron tratamiento neoadyuvante con quimioterapia tuvieron una mediana de edad de 59 años, la mayor parte de ellas presentó un estado funcional adecuado y un nivel de albúmina basal mayor de 3,5 g/dL. Estas características, probablemente permitieron su tolerabilidad al tratamiento sistémico y son similares a los reportado en el estudio realizado por la Organización Europea para la Investigación y Tratamiento del Cáncer (EORTC) ${ }^{(17)}$, donde se ha comparado el tratamiento neoadyuvante con quimioterapia a dosis convencionales seguido de $\mathrm{CCl}$ con la $\mathrm{CCP}$.

La resección completa de la enfermedad macroscópica (CCP o $\mathrm{CCl}$ ) constituye un factor pronóstico independiente de sobrevida global en cáncer de ovario. En 1994, Hoskins et al. describieron que los pacientes con tumores residuales $>2 \mathrm{~cm}$ presentan pobre pronóstico, independiente de la respuesta obtenida a tratamiento neoadyuvante con quimioterapia, no encontrando diferencia en la supervivencia de no alcanzar citorreducción óptima ${ }^{(22)}$.

Estudios retrospectivos y prospectivos indican que el tratamiento neoadyuvante con quimioterapia en el cáncer de ovario avanzado seguido de $\mathrm{CCl}$ aumenta la tasa de citorreducción óptima y no es inferior a la CCP; ya que ha mostrado, una sobrevida libre de progresión y sobrevida global similares ${ }^{(14-16,23,24)}$.

En el presente estudio, se encontró una tasa de citorreducción óptima similar a lo reportado en los ensayos prospectivos fase III como por ejemplo el estudio EORTC ${ }^{(17)}$, el estudio realizado por el grupo clínico oncológico de Japón (JCOG) ${ }^{(18)}$ y el estudio CHORUS ${ }^{(18,25)}$, ellos evaluaron la eficacia de la quimioterapia neoadyuvante con carboplatino y paclitaxel con dosis convencional seguido $\mathrm{CCl}$ comparado con la CCP, y mostraron tasas de citorreducción óptima de $80,6 \%, 72 \%$ y $40 \%$, respectivamente.

Se encontró una mediana de sobrevida libre de progresión de 25 meses, la cual es mayor a lo reportado en otros estudios prospectivos como el EORTC (sobrevida libre de progresión de 12 meses, SG de 30 meses) ${ }^{(17)}$, JCOG (sobrevida libre de progresión 11,7 meses, SG de 24,5 meses) ${ }^{(18)}$ y estudio CHORUS (SLP 24,5 meses, SG de 30 meses) ${ }^{(18,25)}$.

Las variables que resultaron influenciar la SG y sobrevida libre de progresión en los pacientes en estudio fueron el valor sérico de CA 125 postoperatorio y la citorreducción óptima. Estos resultados, corroboran la evidencia de que el nivel sérico de CA 125 en pacientes con cáncer de ovario avanzado es un marcador de respuesta y seguimiento; ya que, los pacientes con valores de CA 125 dentro de límites normales posterior al tratamiento neoadyuvante con quimioterapia, presentan mejor sobrevida ${ }^{(26,27)}$.

Varios estudios han demostrado ampliamente que el tratamiento neoadyuvante con quimioterapia aumenta las tasas de citorreducción óptima en pacientes con cáncer de ovario avanzado y han descrito una sobrevida global a favor del tratamiento neoadyuvante con quimioterapia seguido de cirugía de intervalo, con respecto a CCP. En cuanto al número de ciclos tal como se evidenció en nuestro estudio, tres ciclos de tratamiento neoadyuvante con quimioterapia pueden ser suficientes para lograr citorreducción óptima; administrar tres ciclos o más de tratamiento neoadyuvante con quimioterapia no mostró ventaja en la tasa de citorreducción óptima, por lo que considerando la toxicidad generada al tratamiento sistémico, debemos considerar, la administración de tres ciclos de tratamiento neoadyuvante con quimioterapia como estándar, con el objetivo de no perder la oportunidad de llevar al paciente a cirugía de citorreducción.

Sin embargo, la interrogante sería, si el tratamiento neoadyuvante con quimioterapia seguida de $\mathrm{CCl}$ es superior en eficacia a la CCP, lo cual aún no está determinado. Algunos 
estudios como los mencionados anteriormente ${ }^{(14,17,24)}$ y un metaanálisis que incluyó 21 ensayos clínicos, encontraron que el tratamiento neoadyuvante con quimioterapia seguido de $\mathrm{CCl}$ no tuvo impacto en sobrevida global respecto a la CCP (28); incluso el metaanálisis de Bristow et al. (2006) mostró resultados inferiores con $\mathrm{CCl}{ }^{(29)}$. Una explicación, por la cual el tratamiento neoadyuvante con quimioterapia no mostró un impacto en la sobrevida global fue la inclusión de pacientes con mejor estado funcional y menor carga tumoral en el grupo de pacientes que fueron sometidos a CCP comparado con el grupo que recibió tratamiento neoadyuvante con quimioterapia. Además, los estudios incluidos en el metaanálisis fueron heterogéneos en los años de publicación, el uso de taxanos y la tasa de citorreducción óptima ${ }^{(28)}$

Hasta la fecha, no hay un consenso para definir los criterios de selección para determinar qué pacientes deben recibir tratamiento neoadyuvante con quimioterapia seguido de cirugía de intervalo; por lo que es necesario llevar a cabo una evaluación exhaustiva de selección de pacientes y evitar las complicaciones asociadas al tratamiento neoadyuvante con quimioterapia.

Encontramos un número importante de toxicidades en el grupo de estudio, principalmente las de tipo hematológico como la neutropenia, lo cual pudo estar influenciado por el estado funcional de los pacientes y comorbilidades. Sin embargo, la frecuencia de toxicidad severa, incluido un fallecimiento asociado a quimioterapia fue baja.
En cuanto a las limitaciones del estudio, el diseño retrospectivo podría proporcionar una evaluación no homogénea del estado funcional y la calidad de vida registrada en las historias clínicas. Por otro lado, la selección de los pacientes para la CCP o $\mathrm{CCI}$ dependió de la condición clínica del paciente, hallazgos en laparoscopia o laparotomía y estudios de imágenes. Por otro lado, no se ha determinado el número de cursos de quimioterapia necesarios previo a la cirugía, ni el momento adecuado para realizar $\mathrm{CCl}$.

En conclusión, en pacientes con cáncer de ovario avanzado, el tratamiento neoadyuvante con quimioterapia con carboplatino y paclitaxel a dosis densa seguido de $\mathrm{CCl}$ ha resultado ser un tratamiento exitoso para lograr una tasa alta de citorreducción óptima, y presenta un perfil de toxicidad aceptable en pacientes no elegibles a CCP. A pesar que nuestros resultados son alentadores, debemos considerar un mayor período de seguimiento para determinar el verdadero impacto del tratamiento neoadyuvante con quimioterapia seguido $\mathrm{CCl}$.

Contribución de los autores: Concepción y diseño del estudio: CA, LAML, MOH. Recolección de resultados CA, JGMV, ZMC, JA, MA. Análisis e interpretación CA, JGMV, RMQ, JPO. Redacción del artículo CA, LAML, MOH, ZMC, JGMV. Aprobación de la versión final a publicar LAML, ZMC, MOH, CA.

Financiamiento: Autofinanciado.

Conflictos de intereses: Los autores declaran no tener conflictos de intereses.

\section{REFERENCIAS BIBLIOGRÁFICAS}

1. Ferlay J, Soerjomataram I, Dikshit R, Eser S, Mathers C, Rebelo M, et al. Cancer incidence and mortality worldwide: sources, methods and major patterns in GLOBOCAN 2012. Int J Cancer. 2015 Mar 1;136(5):E359-86.

2. Inciūra A, Juozaityte $E$, Nadisauskiene R, Cigriejiene VM, Kajenas S, Vaitkiene D, et al. [Surgical treatment of advanced epithelial ovarian cancer]. Medicina. 2004;40(3):205-15.

3. Ferlay J, Shin H-R, Bray F, Forman D, Mathers C, Parkin DM. Estimates of worldwide burden of cancer in 2008: GLOBOCAN 2008. Int J Cancer. 2010 Dec 15;127(12):2893-917.

4. Sankaranarayanan R, Ferlay J. Worldwide burden of gynaecological cancer: The size of the problem. Best Pract Res Clin Obstet Gynaecol. 2006;20(2):207-25.

5. A report from the Economist Intelligence Unit. Breakaway: The global burden of cancer- challenges and opportunities [Internet]. London: The Economist Intelligence Unit; 2009 [citado $2018 \mathrm{Feb}$ 18]. Disponible en: http://graphics.eiu. com/upload/eb/EIU_LIVESTRONG_ Global_Cancer_Burden.pdf

6. Eduardo Payet Meza, Patricia Pérez Mejía, Ebert Carlos Poquioma Rojas, Milward Ubillús Trujillo, editor. Registro del cáncer de Lima - Metropolitana 20042005. Vol. IV [Internet]. Lima: Instituto Nacional de Enfermedades Neoplásicas (INEN); 2013. [cited 2018 Feb 18] 217 p. Disponible en: http://www.inen.sld. pe/portal/documentos/04022014 Libro_Reg_Cancer.pdf

7. Instituto Nacional de Enfermedades Neoplásicas. Casos nuevos de cáncer registrados en el INEN, periodo 20002014 (ambos sexos) [Internet]. Lima: departamento de epidemiologia, Instituto Nacional de Enfermedades Neoplásicas (INEN). [cited 2018 Feb 18]. Disponible en: http://www.inen.sld.pe/portal/documentos/pdf/estadistica/datos_estadisticos/02062016_DATOS_EPIDEMIOLOGICOS\%20INEN(2000-2014).pdf

8. Inciura A, Simavicius A, Juozaityte E, Kurtinaitis J, Nadisauskiene R, Svedas E, et al. Comparison of adjuvant and neoadjuvant chemotherapy in the management of advanced ovarian cancer: a retrospective study of 574 patients. BMC Cancer. 2006 Jun 8;6:153.

9. Griffiths CT. Surgical resection of tumor bulk in the primary treatment of ovarian carcinoma. Natl Cancer Inst Monogr. 1975;42:101-4.

10. Lopes NM, Adams EG, Pitts TW, Bhuyan BK. Cell kill kinetics and cell cycle effects of taxol on human and hamster ovarian cell lines. Cancer Chemother Pharmacol. 1993;32(3):235-42.

11. Katsumata N, Yasuda M, Takahashi F, Isonishi $\mathrm{S}$, Jobo $\mathrm{T}$, Aoki $\mathrm{D}$, et al. Dose-dense paclitaxel once a week in 
combination with carboplatin every 3 weeks for advanced ovarian cancer: a phase 3 , open-label, randomised controlled trial. Lancet. 2009;374(9698):1331-8.

12. Katsumata N, Yasuda $M$, Isonishi $S$, Takahashi F, Michimae H, Kimura E, et al. Long-term results of dosedense paclitaxel and carboplatin versus conventional paclitaxel and carboplatin for treatment of advanced epithelial ovarian, fallopian tube, or primary peritoneal cancer (JGOG 3016): a randomised, controlled, open-label trial. Lancet Oncol. 2013;14(10):1020-6.

13. Tan G, Heqing L, Jiangbo C, Ming J, Yanhong M, Xianghe L, et al. Apoptosis induced by low-dose paclitaxel is associated with $\mathrm{p} 53$ upregulation in nasopharyngeal carcinoma cells. Int J Cancer. 2002;97(2):168-72.

14. Everett EN, French AE, Stone RL, Pastore LM, Jazaeri AA, Andersen WA, et al. Initial chemotherapy followed by surgical cytoreduction for the treatment of stage III/IV epithelial ovarian cancer. Am J Obstet Gynecol. 2006;195(2):568-74.

15. Hou J, Kelly M, Yu H, Mcalpine J, Azodi M, Rutherford T, et al. Neoadjuvant chemotherapy lessens surgical morbidity in advanced ovarian cancer and leads to improved survival in stage IV disease. Gynecol Oncol. 2007;105(1):211-7.

16. Vergote I, De Wever I, Tjalma W, Van Gramberen M, Decloedt J, van Dam P. Neoadjuvant Chemotherapy or Primary Debulking Surgery in Advanced Ovarian Carcinoma: A Retrospective Analysis of 285 Patients. Gynecol Oncol. 1998;71(3):431-6.

17. Vergote I, Tropé CG, Amant F, Kristensen GB,Ehlen T,Johnson N, etal.Neoadjuvant Chemotherapy or Primary Surgery in Stage IIIC or IV Ovarian Cancer. N Engl J Med. 2010;363(10):943-53.

18. Onda T, Satoh T, Saito T, Kasamatsu T, Nakanishi T, Nakamura K, et al. Comparison of treatment invasiveness between upfront debulking surgery versus interval debulking surgery following neoadjuvant chemotherapy for stage III/IV ovarian, tubal, and peritoneal cancers in a phase III randomised trial: Japan Clinical Oncology Group Study JCOG0602. Eur J Cancer. 2016;64:22-31.

19. Departamento de Oncologia Médica. Guía de Práctica Clínica de Ovario [Internet]. Lima: Instituto Nacional de Enfermedades Neoplasicas (INEN); 2013 [cited 2018 Feb 19].Disponible en: http:// www.inen.sld.pe/portal/documentos/ pdf/normas_tecnicas/2013/08072014_ G U \% C $3 \% 8$ D A S D E $\mathrm{P} R \%$ C $3 \% 81 \mathrm{C}$ T I C A $\mathrm{C} \mathrm{L} \% \mathrm{C} 3 \% 8 \mathrm{D} N \mathrm{I} C \mathrm{~A}$ DE_C\%C3\%81NCER_DE_OVARIO. pdf

20. NCCN. Ovarian Cancer versión 2. 2018 [Internet]. U.S: National Comprehensive Cancer Network (NCCN); 2018 [citado 2018 Feb 19]. Disponible en: https:// www.nccn.org/professionals/physician gls/pdf/ovarian.pdf

21. U.S. Department of Health and Human Services. Common Terminology Criteria for Adverse Events (CTCAE) v 4.0 [Internet]. U.S. : National Institutes of Health and National Cancer Institute; 2009 [citado 2018 Feb 19]. Available from: https://evs.nci.nih. gov/ftp1/CTCAE/CTCAE_4.03/ C TCAE_4.03_2010-06-14_ QuickReference_8.5x11.pdf

22. Hoskins WJ, McGuire WP, Brady MF, Homesley HD, Creasman WT, Berman $\mathrm{M}$, et al. The effect of diameter of largest residual disease on survival after primary cytoreductive surgery in patients with suboptimal residual epithelial ovarian carcinoma. Am J Obstet Gynecol. 1994;170(4):974-9; discussion 979-80.

23. Vrscaj MU, Rakar S. Neoadjuvant chemotherapy for advanced epithelial ovarian carcinoma: a retrospective case- control study. Eur J Gynaecol Oncol. 2002;23(5):405-10

24. Lee S-J, Kim B-G, Lee J-W, Park C-S, Lee J-H, Bae D-S. Preliminary results of neoadjuvant chemotherapy with paclitaxel and cisplatin in patients with advanced epithelial ovarian cancer who are inadequate for optimum primary surgery. J Obstet Gynaecol Res. 2006;32(1):99-106.

25. Kehoe S, Hook J, Nankivell M, Jayson GC, Kitchener H, Lopes T, et al. Primary chemotherapy versus primary surgery for newly diagnosed advanced ovarian cancer (CHORUS): an open-label, randomised, controlled, non-inferiority trial. Lancet. 2015;386(9990):249-57.

26. Mazzeo F, Berlière M, Kerger J, Squifflet J, Duck L, D'Hondt V, et al. Neoadjuvant chemotherapy followed by surgery and adjuvant chemotherapy in patients with primarily unresectable, advancedstage ovarian cancer. Gynecol Oncol. 2003;90(1):163-9.

27. Le T, Faught W, Hopkins L, Fung-KeeFung M. Importance of CA125 normalization during neoadjuvant chemotherapy followed by planned delayed surgical debulking in patients with epithelial ovarian cancer.J Obstet Gynaecol Can. 2008;30(8):665-70.

28. Kang S, Nam B-H. Does neoadjuvant chemotherapy increase optimal cytoreduction rate in advanced ovarian cancer? Meta-analysis of 21 studies. Ann Surg Oncol. 2009;16(8):2315-20.

29. Bristow RE, Chi DS. Platinum-based neoadjuvant chemotherapy and interval surgical cytoreduction for advanced ovarian cancer: a meta-analysis. Gynecol Oncol. 2006;103(3):1070-6.

Correspondencia: Cindy Alcarraz Molina

Dirección: Av. Angamos Este 2520, Surquillo,

Lima 41, Lima, Perú.

Correo electrónico: cindyalcarraz@gmail.com 